\section{Fourth defence molecule completes antiviral line-up}

\author{
Niklas A. Schmacke \& Veit Hornung
}

Toll-like receptors can initiate an immune response when they detect signs of a viral or microbial threat. New insight into how such receptor activation drives defence programs should aid our efforts to understand autoimmune diseases. See p.316
Cells of the innate branch of the immune system can detect infectious agents outside the cell or in various intracellular compartments. This process depends on the sensing of 'non-self' molecular signatures by proteins known as pattern-recognition receptors

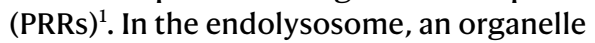
into which extracellular material can be taken up by the cell, PRRs called Toll-like receptor 7 (TLR7), TLR8 and TLR9 can be activated by the presence of viral or microbial nucleic acids $^{2}$. However, these same receptors are often linked to the erroneous detection of 'self' nucleic acids in autoimmune diseases ${ }^{3}$. Heinz et al. ${ }^{4}$ report on page 316 that a protein they name TASL links the activation of TLR7, TLR8 and TLR9 to the production of molecules called type I interferons, which mediate antiviral defence. The gene that encodes TASL has been associated with the autoimmune disease systemic lupus erythematosus, and this finding might shed light on factors that contribute to the disease.

To distinguish between different diseasecausing viruses and microbes and to tailor a suitable response, the innate immune system uses PRRs in various parts of the cell ${ }^{1}$. Each of these sensors recognizes a distinct hallmark of infectious agents termed a pathogenassociated molecular pattern (PAMP). One such family of receptors, the TLRs (Fig. 1), are transmembrane proteins that are found on the cell surface or in the endolysosome ${ }^{2}$. Most cell-surface TLRs detect bacterial components, such as lipopeptides found in bacterial cell walls. By contrast, the endolysosomal TLRs - TLR3, TLR7, TLR8 and TLR9 - recognize nucleic acids or their degradation products, which are typically associated with viral infection but are also a signature of living microbes.

On activation in response to binding a PAMP, TLRs engage another protein, termed an adaptor protein, which provides a crucial control point that sets off distinct signalling cascades culminating in defence responses ${ }^{2}$. Together with other defence mechanisms, two major gene-expression programs can be distinguished that are broadly tailored to the particular threat sensed. Downstream of most TLRs, an adaptor protein called MyD88 activates the transcription-factor protein NF-KB, which drives expression of pro-inflammatory genes as part of the immune response. A subgroup of TLRs (TLR3 and TLR4) can engage the protein TRIF, which acts as a scaffold enabling a kinase enzyme to add a phosphate group to the transcription factor IRF3. This phosphorylation activates IRF3, a member of a family of transcription factors termed interferon regulatory factors (IRFs), which activate broad gene-expression programs. A hallmark of these programs is the production of type I interferon molecules ${ }^{5}$.

Interferons are potent drivers of a branch of the immune system termed the adaptive immune response, and their presence therefore runs the risk of contributing to autoimmunity. To prevent such an attack by the host's own immune system, an interferon response must be tightly regulated. As a safeguard, a particular sequence of amino-acid residues in TRIF, the pLxIS motif, must be phosphorylated before IRF 3 can be activated. This control mechanism provides a 'licensing step' that is not specific just for TRIF as an adaptor protein for TLR signalling, but is a general hallthe related protein IRF7, to drive interferon expression. Every identified innate sensing pathway connecting the recognition of nucleic acids to the production of type I interferons, with one exception, had been shown previously to signal through one of the three adaptor proteins known so far to contain a pLxIS motif: TRIF, MAVS and STING. Thus, pLxIS-motif-containing adaptor proteins specifically hardwire nucleic-acid recognition to antiviral defences.

The only exception to this rule had been the endolysosomal TLRs - TLR7, TLR8 and mark of sensing pathways that engage IRF3, or

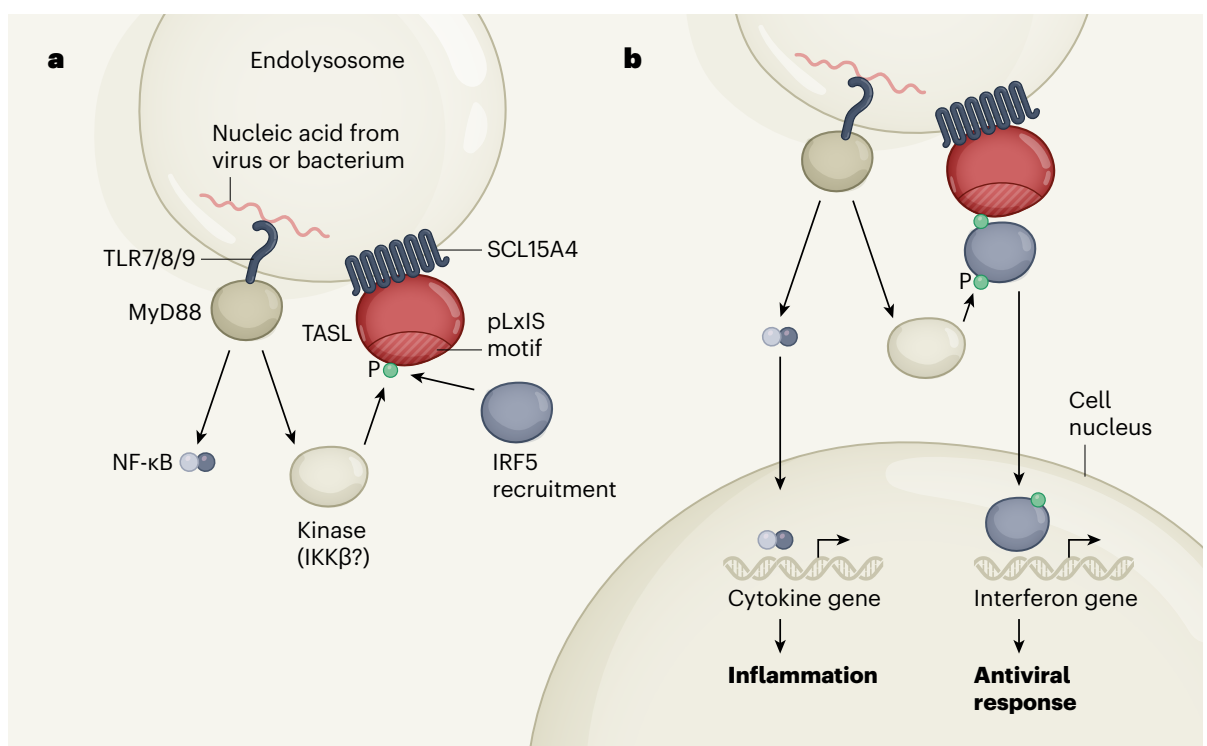

Figure $1 \mid$ A crucial role for the TASL protein in the activation of interferon-mediated antiviral defences. a, Nucleic acids from viruses or bacteria can be taken up by human immune cells into an organelle called the endolysosome and are recognized by Toll-like receptor proteins (TLR7, TLR8 and TLR9). This recognition activates the protein MyD88, which, in turn, activates the transcription-factor protein NF-KB, a key player in the immune response to infection. Heinz et al. ${ }^{4}$ investigated how a protein called SCL15A4, which is associated with the autoimmune disease systemic lupus erythematosus, aids this defence response. They found that it binds to a protein named TASL, which contains an evolutionarily conserved amino-acid sequence called the pLxIS motif. When endolysosomal TLRs recognize foreign nucleic acids, a phosphate group (P) is added to TASL by a kinase enzyme (possibly IKK $\beta$ from a pathway downstream of MyD88). This phosphorylation recruits the transcription factor IRF5 to TASL. b. TASL then acts as a scaffold to facilitate the phosphorylation and activation of IRF5 by a kinase (possibly IKK $\beta$ ). It is the first pLxIS-containing protein known to mediate IRF5 activation. Phosphorylated IRF5 enters the nucleus and drives the expression of genes that encode antiviral interferon molecules. NF-KB drives the expression of pro-inflammatory defence molecules called cytokines. 
TLR9. Although these detect nucleic acids and drive type I interferon gene expression, they do not use TRIF (ref. 2). Instead, they use MyD88 to activate the interferon regulatory factor IRF5 (ref. 6), which is related in structure and function to IRF3 and IRF7. A licensing step involving a pLxIS motif had not been found previously in the signalling cascades of TLR7, TLR8 and TLR9.

Immune cells called plasmacytoid dendritic cells express high levels of TLR7 and TLR9, and are crucial to antiviral defences through their production of large amounts of type I interferon. But these cells are also central players in systemic lupus erythematosus ${ }^{7}$. Previous studies of the molecular mechanisms underlying this disease $\mathrm{e}^{7,8}$ have identified a protein called SLC15A4, located on endolysosomal membranes, which transports polypeptides and the amino acid histidine. SLC15A4 has been linked to the activation of TLRs.

To investigate the role of SLC15A4 further, Heinz and colleagues used mass spectrometry to probe for proteins that interact with it. This approach identified the protein TASL, which had previously been little researched. TASL is highly abundant in cells of the innate immune system, and Heinz and colleagues found that it is tethered to endolysosomes through interactions with SLC15A4. The authors' further experiments confirmed that this interaction is specific: SLC15A4 bound TASL in immunoprecipitation tests; however, neither the related protein SLC15A3 nor a mutant version of SLC15A4 interacted with TASL in such assays.

When the authors engineered plasmacytoid dendritic cells and immune cells called monocytes to lack expression of the gene encoding TASL, they found that signalling mediated by TLR7, TLR8 and TLR9 was abolished, and a similar effect was seen when SLC15A4 was absent. Heinz et al. went on to demonstrate that TASL acts specifically through IRF5 by finding that the response to TLR7 and TLR9 activation remained intact in immune cells lacking IRF3 or IRF7, but was blocked in cells deficient in TASL or IRF5. However NF-KB-mediated signalling was unaffected when the pathway acting through TASL was disrupted. Intriguingly, the authorsidentified a pLxIS motif in TASL, and found evidence that phosphorylation of this motif - by kinases downstream of MyD88 that are associated with NF-KB activation - mediates IRF5 activation.

This discovery elevates TASL to membership of an exclusive circle of IRF-activating adaptor proteins containing pLxIS motifs, of which the other members are TRIF, MAVS and STING (ref. 5). These four proteins together control the type I interferon response induced by nucleic-acid sensing, a picture that has now been completed with the discovery of TASL as the missing pLxIS adaptor of TLR7, TLR8 and TLR9 signalling.
Given that TASL signals to IRF5, but not to IRF3 or IRF7, it will be interesting to determine the structural features required for the differential recruitment of IRF-family members to pLxIS-motif-containing proteins. Although the authors performed preliminary experiments to investigate phosphorylation events in this system, phosphorylation of the pLxIS motif in TASL should be investigated in detail to identify the kinase(s) responsible.

Moreover, it will be interesting to sort out how this newly identified signalling pathway operates in relation to activation of the pathway involving MyD88, which is the key adaptor of TLR7, TLR8 and TLR9 signalling 9 . As has been shown for other signalling cascades triggered by TLRs and involving multiple adaptors, it is possible that MyD88-mediated signalling and the pLxIS licensing step involving TASL emanate sequentially from distinct endolysosomal vesicles at different stages of maturation ${ }^{2}$. Although TASL is not involved in NF-KB activation, the authors found that the expression of certain pro-inflammatory genes was still blocked in TASL-deficient cells, probably because of the associated defect in IRF5 activation. Nonetheless, by offering a way to dampen interferon-mediated autoimmunity in a way that doesn't block the ability to launch an inflammatory defence response, TASL might prove to be a drug target for treating autoimmune diseases that are fuelled by the engagement of TLR7, TLR8 and TLR9.

Niklas A. Schmacke and Veit Hornung are in the Department of Biochemistry and at the Gene Center, Ludwig-Maximilians-Universität München, Munich 81377, Germany. e-mail: hornung@genzentrum.lmu.de

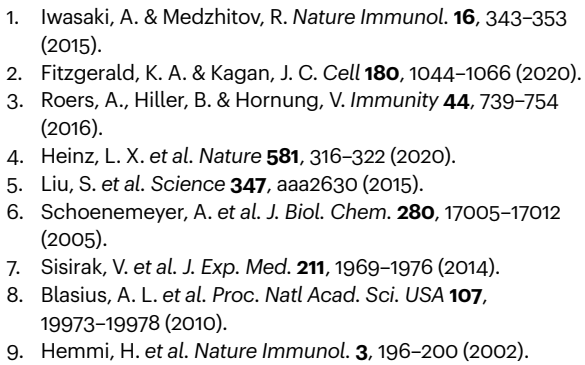

This article was published online on 13 May 2020.

\section{Astronomy}

\section{An early start for galactic disks}

Alfred Tiley

A powerful radio telescope has peered back through time to observe a galaxy that contained a cold, rotating disk of gas not long after the Big Bang - fuelling the debate about when and how disks first formed in galaxies. See p.269

Galaxies are immense, gravitationally bound systems composed of stars, dust, gas and invisible 'dark matter'. Understanding how galaxies have formed and grown over time is essential for a more general view of how matter assembles into large structures - a key piece of the puzzle in our efforts to comprehend the Universe. A crucial step towards this goal is to obtain a clear picture of when disk structures first appeared in galaxies. On page 269 , Neeleman et al. ${ }^{1}$ present observations that reveal a massive, rotating disk of cold gas inside a star-forming galaxy only 1.5 billion years after the Big Bang. This is considerably earlier in cosmic history than the times when previously detected gas disks were found to have existed ${ }^{2}$.

According to our current understanding of cosmology, the earliest large-scale structures in the Universe were spherical dark-matter 'haloes' that collapsed under their own gravity ${ }^{3}$. Surrounding gas fell into these haloes, subsequently forming stars and, ultimately, galaxies ${ }^{4}$. Haloes and galaxies are thought to have continued to grow together by hierarchical assembly (merging), and through the further accretion of gas and its conversion to stars ${ }^{5}$. Hierarchical assembly is simple, and is thought to be well understood. However, there is still much debate surrounding the exact pathways by which gas accretion and its assembly into stars occurs, and how it relates to the formation of physical and dynamical structures in galaxies over time.

A key component of this mystery is why some galaxies, such as our own star-forming Milky Way, have physical structures dominated by disks of stars and gas (Fig. 1), whereas other, generally older and more quiescent galaxies do not. The answer is probably 